\title{
Politische Persuasion im europäischen Parlament: Deutsch-Italienisch im Vergleich
}

\author{
Lucia Cinato (Torino)
}

\begin{abstract}
What strategies do politicians use to convey their ideas and convince the audience of what they say? How are these devices received by interpreters and, above all, how are they interpreted? In my article, I will use some speeches delivered by German-speaking members of the European Parliament to examine how the language of politics and the persuasion strategies of politicians are expressed and how simultaneous interpreting can reproduce these strategies. In the latter, due to temporal and other constraints, not all elements of the original oral text can be reproduced, such as wordplay, word formation and rhetorical figures at the lexical level and information structure at the syntactic level. If the oral speeches are then reproduced in written form, as is usual at the European Parliament, certain content and language strategies can be recovered, but only in this later phase, which definitely loses some features of the spoken language. The question to be investigated in this article is: is it possible to reproduce, in a convincing manner, the message of a source text even if elements of persuasion strategies are missing? Pragmatic, textual and linguistic-system considerations will illustrate the theoretical framework with the aid of concrete examples
\end{abstract}

\section{$1 \quad$ Einleitung}

Der vorliegende Beitrag will einerseits persuasive Kommunikationsstrategien von Politikern untersuchen und andererseits Möglichkeiten und Grenzen einer Reproduktion ebendieser Strategien in Simultanverdolmetschungen nachgehen ${ }^{1}$ Dass beim Simultandolmetschen aus zeitlichen und inhaltlichen Gründen ${ }^{2}$ nicht immer alle Elemente des originalen mündlichen Textes wiedergegeben werden, ist offensichtlich, besonders bei Sprachspielen, Wortbildungen sowie rhetorischen Figuren auf lexikalischer Ebene und Informationsstruktur auf syntaktischer Ebene. ${ }^{3}$ Werden die mündlichen Redebeiträge schriftlich wiedergegeben, wie im euro-

\footnotetext{
${ }^{1}$ Unter Simultanverdolmetschungen (hier auch als Simultandolmetschen oder Simultanverdolmetschen bezeichnet) versteht man die mündliche Übertragung von gesprochenen Texten in andere Sprachen. Beim Simultandolmetschen befinden sich die Dolmetscher in einer schallisolierten Dolmetscherkabine, wo sie das Gesagte über Kopfhörer hören und es simultan (mit einem kleinen zeitlichen Versatz) in die andere Sprache übertragen. Da Simultandolmetschen höchste Konzentration erfordert, wird in der Regel mit einem Team aus zwei Dolmetschern gearbeitet, die sich alle 20-30 Minuten abwechseln und sich gegenseitig unterstützen.

2 Z. B. die Notwendigkeit der Textverdichtung, d. h., redundante Elemente weglassen.

${ }^{3}$ Nach Schreiber (2012: 86) arbeiten Übersetzer immer (in der Regel implizit) mit einer „Hierarchie der Invarianzforderungen“, da in einer Übersetzung selbstverständlich nie alle Elemente des Originals erhalten bleiben
} 
päischen Parlament üblich, können bestimmte Inhalte und Sprachstrategien in der schriftlichen Übersetzung zurückgewonnen werden. Durch die Verschriftlichung, die erst in einer späteren Phase erfolgen kann, gehen allerdings einige Merkmale der gesprochenen Sprache verloren.

In diesem Beitrag sollen Funktion und Relevanz von bestimmten strategischen Sprachverhaltensweisen für die Vermittlung der Handlungsabsicht untersucht werden. In diesem Zusammenhang gilt es auch, der Frage nachzugehen, inwiefern die Botschaft des Ausgangstextes (AT) in Simultanverdolmetschungen in überzeugender Weise wiedergegeben werden kann. ${ }^{4}$ Im Folgenden werden pragmatische sowie text- und systemlinguistische Betrachtungen anhand von konkreten Beispielen vorgestellt. Besondere Aufmerksamkeit wird den Methoden persuasiver Einwirkung (cf. Golonka 2009) und der Emotionalisierung als persuasive Strategie (cd. Schwarz-Friesel 2007) gewidmet.

\section{Korpus}

Bei dem vorliegenden Korpus handelt es sich um eine Pilotstudie, bestehend aus 10 kurzen Reden, die zwischen 2009 und 2011 während verschiedener Plenarsitzungen im Europäischen Parlament von deutschsprachigen abgeordneten Frauen zum Thema Gleichstellung und Gleichbehandlung von Männern und Frauen vorgetragen wurden, sowie deren Verdolmetschung ins Italienische. ${ }^{5}$ Das Thema wurde vor allem wegen seiner zeitlosen Aktualität und seiner für alle Parteien und Gruppen hohen Relevanz gewählt. Es handelt sich um sogenannte Aussprachen während der Plenartagung im Europäischen Parlament, die mit Anfragen an Rat und Kommission verbunden sind und eine Länge von ungefähr einer bis anderthalb Minuten aufweisen. Aufgrund der Politik der Mehrsprachigkeit können die Abgeordneten ihre Reden in Ausschuss- und Plenarsitzungen sowie in Fraktions- und Arbeitsgruppensitzungen in jeder der 24 offiziellen Amtssprachen halten, eine Simultandolmetschung wird gewährleistet. ${ }^{6}$ Für eine genaue Analyse der Originalreden und deren Verdolmetschung reichen allerdings die offiziellen Niederschriften nicht aus. Der Grund dafür ist, dass in den offiziell redigierten Verschriftlichungen des Europäischen Parlaments Oralitätsmerkmale fehlen: Beispielsweise werden typische syntaktische Strukturen der gesprochenen Sprache, die gegen die standardsprachliche Norm verstoßen, weggelassen oder korrigiert. Außerdem sind die Simultan-

\footnotetext{
können. „Bei politischen Reden ist zu bedenken, dass stilistische Elemente wie Metaphern und Wiederholungen kein Selbstzweck sind, sondern im Dienste einer höherrangigen Invariante, nämlich der Textfunktion (z. B. der 'Persuasion') stehen. Es wäre somit sicher verfehlt, von einer Übersetzung zu verlangen, alle Metaphern und Wiederholungen so wörtlich wie möglich nachzubilden, ohne dabei zu beachten, ob sich dies negativ auf die Erhaltung der Textfunktion auswirkt, z. B. wenn eine wörtliche Übersetzung übertrieben pathetisch in der Zielkultur wirken würde“ (ibd.). In einer früheren Arbeit hatte Schreiber (1993: 125) Übersetzungen von Bearbeitungen unterschieden: Übersetzungen beruhen auf einer einzigen Varianzforderung (der Forderung nach Änderung der Sprache) und ansonsten ausschließlich auf Invarianzforderungen, während Bearbeitungen auf einer Invarianzforderung (der Forderung nach Beibehaltung mindestens eines individuellen Textmerkmals) und ansonsten ausschließlich auf Varianzforderungen basieren. Letzteres könnte auch für das Dolmetschen gelten, da der Dolmetschprozess viele Ähnlichkeiten mit dem Bearbeitungsprozess aufweist.

${ }^{4}$ In Bezug auf Translationsstrategien beim Simultandolmetschen cf. Cinato (2016).

5 Siehe „Corpus" im Literaturverzeichnis.

6 ,Mehrsprachichkeit im europäischen Parlament“.
} 
dolmetschungen nur als mündliche Texte verfügbar, da sie juristisch keinen Wert besitzen. Die offiziellen Übersetzungen werden wiederum auf der Basis der offiziellen Verschriftlichungen angefertigt, so dass nicht selten Widersprüchlichkeiten zu den mündlichen Texten entstehen. Überdies werden sie oft aus dem Englischen übersetzt, was ebenfalls zu Abweichungen vom Original führen kann. Für diese Studie wurden deshalb sowohl die Reden als auch deren Simultandolmetschungen transkribiert ${ }^{7}$, um den original gesprochenen Texten möglichst nahe zu bleiben. Selbst bei einer originalgetreuen Transkription der Texte gehen dennoch viele Elemente der Mündlichkeit verloren, so z. B. nonverbale Mittel (Mimik, Gestik, Körperhaltung und vokale Zeichen), die unabdingbar für eine Gesamtanalyse der Merkmale gesprochener Sprache wären, in dieser Studie aber nicht berücksichtigt werden können.

Als Textsorte der politischen Kommunikation hat die parlamentarische Rede und damit auch jede Rede innerhalb des Europäischen Parlaments das Ziel, eine parlamentarische Debatte in Gang zu setzen, d. h. sie ist von Natur aus so konzipiert, dass daraus eine Plenardiskussion über ein bestimmtes Thema entsteht. Jeder Einzelredner einer beliebigen Partei versucht, die Plenarsitzung positiv für sich zu beeinflussen, sie zu behaupten. Die daraus entstehende Debatte ist durch eine Auseinandersetzung zwischen den Abgeordneten charakterisiert, die zuerst monologisch formuliert, und danach durch eine direkte Gegenrede des Plenums und festgelegte Rednerrollen gekennzeichnet wird, wobei die Beiträge nicht immer frei gesprochen werden, sondern oft das Ergebnis einer vorausgeplanten Formulierung sind. Die politische Rede ist nur sehr selten eine improvisierte Rede, sie unterliegt normalerweise verschiedenen Mustern, die viel Vorbereitung benötigen und nicht nur der Diskussion sondern auch den Positionen der verschiedenen Parteien und Fraktionen verpflichtet sind. Bei dem untersuchten Korpus kommt noch hinzu, dass die Zeit der Beiträge auf maximal eine bis zwei Minuten begrenzt ist, was dazu führt, dass vorgelesene Texte den Rednerinnen erlauben, in kurzer Zeit mehr zu sagen als in Stegreif-Reden. Von den zehn Beispielreden wird nur eine frei gesprochen, alle anderen werden vom Blatt gelesen. Das Blatt, was die Rednerinnen vor Augen halten, ist nicht unbedingt ein vollständiger Text, es könnte sich auch nur um Stichwörter oder Notizen oder eine Gliederung handeln, in jedem Fall um schon im Voraus reflektierte Inhalte. Die Reden, die vor dem Parlament gesprochen werden, sind keine spontanen Reden aber auch keine Texte tout court, man kann es eher als „formelles Sprechen“ bezeichnen: schriftlich konzipiert aber mündlich realisiert (cf. Gutenberg 2000). Politische Redetexte sind also spezifische Formen des Sprachgebrauchs; ihre Wirkungsintention entfaltet sich im Zusammenspiel von Redner, Plenarsaal, Text, Intention und persuasiven Mitteln.

\section{Herausforderungen beim Simultandolmetschen im Europäischen Parlament}

Zu den Hauptschwierigkeiten, mit denen sich der Dolmetscher zwar eigentlich immer, aber in der EU besonders stark konfrontiert sieht, gehört sicherlich der Zeitfaktor, sein Hauptfeind. Der Dolmetscher darf den Informationsfluss nie unterbrechen, kann aber gleichzeitig den Sinn der Äußerung nicht (immer) antizipieren. Somit befindet er sich stets in einer Stresssituation, weil sich bei ihm rezeptive und produktive Phasen überlappen. Die erworbene Technik hilft

\footnotetext{
${ }^{7}$ Für die Transkription dieser Reden und deren entsprechenden Verdolmetschungen wurde hier auf ein spezifisches Transkriptionssystem verzichtet, da Konversations- und Diskursanalyse sowie Interaktionale Linguistik, für welche spezifische Transkriptionssysteme unabdingbar sind, nicht im Mittelpunkt dieser Studie stehen.
} 
ihm allerdings, die Stresssituation zumindest teilweise im Griff zu haben. Hinzu kommt auch noch die oben erwähnte Tatsache, dass die Redner selten frei sprechen, sondern meistens vorlesen, was zu einem erhöhten Redetempo und zum verringerten Vorkommen von den der Mündlichkeit inhärenten Pausen und Häsitationssignalen führt (cf. Brambilla 2007). In einigen Fällen bekommt der Dolmetscher die Notizen der Redner im Voraus, was natürlich seine Aufgabe erleichtert. Der Dolmetscher sollte in der Lage sein, sowohl die Textsorten als auch ihre typisch wiederkehrenden Merkmale sofort zu erkennen, wie z. B. Begrüßungs- und Danksagungsformeln, sodass deren Wiedergabe zum Automatismus wird und er sich auf das Neue im Text konzentrieren und seine Energie für die passenden Strategien einsetzen kann. Die Anwendung von generellen und spezifischen Strategien für sein Sprachenpaar befreit den Dolmetscher nicht von den vielen Schwierigkeiten, die er allein in Angriff nehmen muss. Der Redner nimmt ihn wegen der räumlichen Distanz oft gar nicht als Interaktionselement wahr, sondern eher als eine Maschine, auch wenn das im Europäischen Parlament nicht uneingeschränkt gilt, da die Rolle und die Bedeutung der Dolmetscher in der Regel anerkannt sind. Was der Dolmetscher zum Teil entscheiden kann, ist der sogenannte décalage, d. h. der zeitliche Abstand (time lag) zum Redner selbst, der sowohl von den Textsorten und dem Sprachenpaar als auch von den psycho-physischen Konditionen des Dolmetschers abhängt.

Vorhergehende Untersuchungen (cf. Cinato 2016: 246-247) haben gezeigt, dass die meisten parlamentarischen Reden den schriftsprachlichen Syntaxregeln folgen, Wiederholungen vermeiden oder sie bewusst aus stilistisch-persuasiven Gründen benutzen und dass sie wohlgeformte Sätze verwenden. Sie können aber auch Merkmale gesprochener Sprache aufweisen: plötzliche Abbrüche (Aposiopesen), bestimmte Diskursstrategien, rituelle Formeln mit verba dicendi oder sentiendi, Füllwörter, Fehlstarts, die einleitende Konjunktion und, Verbesserungen, freistehende Nebensätze, Spaltsätze, Anakoluthe, Ausklammerungen und präzisierende Nachträge, Redundanzen, Reparaturen oder auch prosodische Elemente wie Pausen und Wiederholungen, also Mittel, die in einer Rede für bestimmte Zwecke, nicht zuletzt aus persuasiven Gründen, verwendet werden.

Die parlamentarische Rede hat somit eine doppelte, hybride Natur zwischen der geschriebenen und der gesprochenen Sprache (cf. Brambilla 2007). Das macht sie besonders forschungswürdig, weil sie als eine eigenständige Textsorte betrachtet werden kann, die zudem innerhalb des Europäischen Parlaments einige Spezifika aufweist, wie im nächsten Kapitel erläutert wird.

\section{Die Textsorte ,parlamentarische Rede“ im Europäischen Parlament}

Bei jeder politischen Rede geht es, neben der informativen Funktion, vor allem um das Überzeugen und Überreden (persuasive Funktion). ${ }^{8}$ Hauptziel der politischen Sprache ist die Konsensstiftung beim Empfänger/Bürger. Das Europäische Parlament (EP) stellt einen besonderen Fall dar: Im Unterschied zu den nationalen Parlamenten (mit Ausnahme von Kanada und der Schweiz) kann der kritische Meinungsaustausch im EP nur stattfinden, wenn mehrere Dolmetscher anwesend sind, die die Redebeiträge verdolmetschen. Das Parlament ist eines der wenigen Organe, wahrscheinlich das einzige Organ innerhalb der Europäischen Union

\footnotetext{
8 Zur Problematik der Dichotomie von „Überzeugen“ und „Überreden“cf. Ortak (2004).
} 
(EU), welches das Prinzip der Mehrsprachigkeit bewahrt und den Vertretern der Mitgliedsstaaten die Benutzung der eigenen Muttersprache gewährleistet. Damit trotz der vielen Sprachen Kommunikation stattfinden kann, müssen Simultandolmetscher eingesetzt werden. Die Kommunikation, die so entsteht, ist eine ,vermittelte“ Kommunikation, ${ }^{9}$ in der der Dolmetscher als Sprachmittler die Interaktion zwischen den Teilnehmern ermöglicht (cf. zu diesem Aspekt Cinato 2018). Ohne ihn würde keine Debatte stattfinden, weil viele Redner nur ein sehr begrenztes Publikum ansprechen würden. Bemerkenswert ist die Konstellation und die räumliche Positionierung der Akteure, die an der Interaktion im EP teilnehmen: Die Redner befinden sich im Plenarsaal, die Dolmetscher in der Kabine und die Zuhörer sitzen ebenfalls im Plenarsaal, sind mit dem Dolmetscher aber durch einen Kopfhörer verbunden. Obwohl sich der Dolmetscher räumlich außerhalb der Interaktion befindet, trägt er aktiv zur Debatte bei und ist derjenige, der entscheidet, was und wie zu übertragen ist. Eine direkte Rückkopplung von den Zuhörern bleibt meistens aus, auch wenn das Publikum den Redner und das Thema im Europaparlament oft kennt und sich zur Qualität des Dolmetschens zeitversetzt aber nur selten direkt äußern kann. All diese Faktoren werden genannt, weil sie mit der Wiedergabe von Persuasionsstrategien direkt zu tun haben: Bestimmte Elemente, die bei einer Rede vor einem Publikum eine sehr große Rolle spielen, werden zweitrangig, weil der Dolmetscher erstens in einer Kabine sitzt und zweitens in kurzer Zeit viel verdolmetschen muss. Gemeint sind nicht nur der Verlust von Augenkontakt und anderen Faktoren wie Körperhaltung, Ton usw., sondern auch viele Ausdrücke, die für das Verstehen einer Rede nicht essentiell sind. Die sich daraus ergebenden notwendigen Modifikationen betreffen vor allem Umstellungen und Tilgungen sowohl auf der makrostrukturellen als auch auf der mikrostrukturellen Ebene der Reden. Ganze Sätze und Satzteile, Sequenzen, einzelne Lexeme usw. werden ausgelassen - alles, was für die Realisierung der Botschaft in der Zielsprache nicht nötig ist. Es handelt sich hauptsächlich um kürzende Eingriffe, die oft auch die Persuasionsstrategien betreffen.

\section{Die Wiedergabe von Persuasionsstrategien beim Simultandolmetschen}

Die Persuasion ist ein wichtiger Teil der zwischenmenschlichen Kommunikation und kann nach Lewandowski (1994: 790) als „Symbolmanipulation mit der Absicht, bei den Adressaten ein bestimmtes Verhalten auszulösen“ definiert werden. Sie ist also „Prozess oder Methode der Beeinflussung von Menschen hinsichtlich ihrer Meinung, ihrer Einstellung sowie ihres Handelns und wendet sich vor allem an die Emotionen der Adressaten" und realisiert sich besonders in einer direkten face-to-face-Kommunikation oder in der Massenkommunikation (Golonka 2009: 161). Die persuasive Intention wird durch eine appellative Textentfaltung ausgedrückt, sie wird sorgfältig geplant und ist durch einen möglichst effektvollen Einsatz sprachlicher und/oder nicht sprachlicher Mittel gekennzeichnet. Unter den sprachlichen Mitteln sind rhetorische Figuren, indirekte Sprechakte, semantisch dichte Ausdrücke oder versteckte Präsuppositionen hervorzuheben, während unter den nicht sprachlichen Mitteln visuelle und auditive Zeichen zu nennen sind (cf. hierzu Golonka 2009: 161-162). Selbst wenn man sich aber nur auf die sprachlichen Mittel der Persuasion konzentrieren möchte und vor allem auf deren Wiedergabe in einer anderen Sprache, ist es nicht immer eindeutig, sie als solche

\footnotetext{
${ }^{9}$ Der Terminus stammt von Kade (1977).
} 
ausfindig zu machen: Welche sprachlichen Mittel dienen tatsächlich der Persuasion? Auf welche Strategien soll die Übertragung achten? Der Überzeugungskraft sind Elemente wie Auswahl der grammatischen Kategorien, semantische Struktur, Wortwahl, Stil und Varietät, syntaktische Anordnung, Textaufbau sowie Benutzung bestimmter Argumentationsstrategien untergeordnet (cf. Golonka (2009: 163). Für den Redner gibt es sehr viele Mittel, die dazu dienen, persuasive Zwecke zu erreichen und die große Unterschiedlichkeit an möglichen Strategien erleichtert die kontrastive Analyse sicher nicht. Nichtsdestotrotz soll in den nächsten Abschnitten versucht werden, einige Strategien der Persuasion auf der Makro- und Mikroebene des Diskurses zu erkennen, um die Simultandolmetschung zu analysieren und ihre entsprechende mögliche Wirkung zu untersuchen.

\subsection{Makroebene des Diskurses}

Um die folgenden Beispiele besser zu verstehen, soll hier zunächst eine kurze inhaltliche Erklärung zum Thema des Korpus gegeben werden. Das Thema ist überparteilich und die Absicht aller Abgeordneten (in diesem Fall ausschließlich Frauen) ist es, zu zeigen, dass es trotz vieler Fortschritte in der Frage der Gleichberechtigung/Gleichbehandlung zwischen Männern und Frauen sowohl in Europa als auch in anderen Teilen der Welt noch viel zu tun gibt. Die Diskussion im Plenum verläuft quer durch die Fraktionen, Zwischenrufe bleiben aus, Applaus und Zwischenapplaus gibt es von allen Seiten und der politische Gegner scheint die Kommission und/oder der Rat selbst zu sein, die noch nicht genug tun, um die Gleichstellung der Frauen tatsächlich zu ermöglichen. Das beweist die Tatsache, dass Frauen in Führungspositionen, an der Spitze von Wirtschaftsunternehmen aber auch von europäischen Institutionen noch immer selten sind. Die kommunikative Absicht wird nach einer kurzen Einführung, die meist Begrüßungsformeln (Herr/Frau Präsident-in, Herr/Frau Vorsitzende, Sehr geehrter Herr Kommissar/Frau Kommissarin, Meine Damen und Herren, Liebe Kolleginnen und Kollegen) oder Danksagungsformeln beinhaltet, mittels Argumentation erzielt. Die Struktur aller Reden ist mehr oder weniger die gleiche: anreden, informieren, legitimieren durch Argumentieren, appellieren. Die Sprache dieser Reden unterscheidet sich sowohl von der Gemeinsprache, weil sie viele Fachwörter beinhaltet, als auch von einer spezifischen Fachsprache. Sie ist eher ein Konglomerat von verschiedenen Fachsprachen: von dem spezifischen Wortschatz der Europäischen Union hin zum Wortschatz der Arbeitsbeschäftigung, der sozialpolitischen Maßnahmen, der Frauenpolitik usw.

\subsubsection{Strategien der persuasiven Einwirkung auf Diskursebene}

Im Folgenden werden zunächst die allgemeinen Persuasionsstrategien auf der Ebene des Diskurses $^{10}$ und ihre Verwendung im Korpus untersucht. Des Weiteren (5.1.2) werden Strategien dargestellt, die allgemeine Überredungsstrategien sind, aber schon die Ebene der Sprache betreffen und mit der Rolle der Emotionalisierung zu tun haben. Schließlich werden unter Punkt 5.2 einige spezifische sprachliche Mittel der Persuasion und deren Verdolmetschung analysiert.

\footnotetext{
${ }^{10}$ Ich benutze den Begriff „Diskurs“ im Sinne von Schröter/Björn 2009, die die politische Rede in Wort (Wortschatz der Politik), Text (politikspezifische Kommunikationsformen) und Diskurs (übergreifende interaktionale Zusammenhänge) unterscheiden.
} 
Ganz allgemein kann man nach Golonka (2009: 161) vier Strategien persuasiver Einwirkung unterscheiden:

1. Emotionalisierung und gefühlsbetonte Ansprache, die eine stark emotionalisierte Aufnahme sichern sollen. Typisch sind hier Vagheit, Bezugnahme auf Sympathien, Vorurteile und Stereotypen, Bewertungen. Die Gefühlsansprache fördert eine unreflektierte Rezeption der kommunizierten Inhalte, begünstigt Übernahme und Festigung bestimmter Wertungen und Urteile. Emotionen sind von Natur aus subjektiv. Ihre Subjektivität kann dazu benutzt werden, falsche, nicht verifizierbare oder aber mit dem eigentlichen Thema nicht verbundene Inhalte zu vermitteln. Neben sofortigen Wirkungen kann emotionale Ansprache auch spätere Folgen haben.

Golonka (2009: 161)

Im Korpus werden oft Bewertungen der Situation der Frauen vorgenommen, die wegen der Arbeitsbedingungen, der Lohnungleichheit, sozialer und gesellschaftlicher Schwierigkeiten usw. als noch sehr mangelhaft dargestellt wird. Auch einige Gesetze, die anscheinend zum Vorteil der Frauen verabschiedet wurden, schaden letztendlich den Frauen selbst, z. B. die Debatte um den Mutterschutz, wo die Sonderstellung der Frau zur Folge hat, dass Frauen zu teuer sind und letztendlich nicht eingestellt werden. Außerdem sind Frauen wegen Teilzeitbeschäftigung, niedrigeren Löhnen, Arbeits- und Einstellungsbedingungen, prekären Arbeitsverhältnissen, sozialer Sicherheit, Kinder- und Altersbetreuung usw. immer noch benachteiligt und dem Armutsrisiko ausgesetzt. Hier unten zwei Beispiele. ${ }^{11} \mathrm{Im}$ ersten Beispiel plädiert Frau Rebecca Harms aus Anlass des 100. Jahrestages der Frau für echte Gleichberechtigung zwischen Männern und Frauen, die auch mittels neuer Gesetze und Regeln, darunter z. B einer Frauenquote, zu erreichen ist. In dem zweiten schildert die Abgeordnete Christa Klaß die noch ungerechte Situation zwischen Männern und Frauen im Arbeitsleben in Europa ${ }^{12}$ :

$\mathrm{DE}$

(1) Herr Präsident, meine Damen und Herren! Ich finde, dass eigentlich wir eine Situation vorfinden, die nicht danach ruft, einen Feiertag durchzuführen, sondern die danach ruft, sich neu zu verabreden zur Verwirklichung echter Gleichberechtigung. Gerade am 100. Jahrestag, ja, sollten wir als Frauen zurückblicken und bilanzieren.

(2) Frau Präsidentin, Herr Kommissar, meine Damen und Herren! Wir stellen heute zum wiederholten Male fest, dass Frauen in Europa noch immer nicht gleichberechtigt im Arbeitsleben sind. Zwar ist die Erwerbstätigenquote von
IT

Presidente, colleghi, ma questa situazione non ci deve portare soltanto alla celebrazione, ci deve invitare, ecco, ad applicare veramente la pari opportunità. Proprio per il centesimo anniversario di questa festa noi dovremmo guardarci indietro e fare un bilancio.
Grazie signora Presidente, signor Commissario, signore e signori, per l'ennesima volta constatiamo che le donne in Europa non hanno nella vita del lavoro la parità. 51 per cento nel 97 al 58 per cento nel 2009 [sic] significa che

\footnotetext{
${ }^{11}$ Alle Beispiele geben die Reden genauso wieder, wie sie tatsächlich ausgesprochen wurden, also auch mit Fehlern nicht nur in der Verdolmetschung, sondern manchmal auch in der Ausgangssprache.

12 Die Hervorhebungen sind durch die Autorin erfolgt. Die Textnummer bezieht sich auf die Nummerierung des Corpus im Literaturverzeichnis.
} 
$51 \%$ in 1997 auf 58\% in 2007 gestiegen. Frauen sind heute hochqualifiziert, der Anteil weiblicher Führungskräfte ist aber in den letzten Jahren unverändert geblieben - unverändert niedrig. Dabei ist der Anteil weiblicher Studierender gerade in den Studienrichtungen Wirtschaftswissenschaften, Betriebswirtschaft und Rechtswissenschaften höher als der der männlichen Studierenden. sono più diplomate però il loro tasso di occupazione è rimasto ugualmente basso. Sono diplomate nelle scienze economiche, nella giurisprudenza, nelle scienze politiche e il loro numero è sempre maggiore dei diplomati uomini.

Die Zielsprachenversionen zeigen, dass es oft zu Abweichungen vom Originaltext kommt. Im Beispiel (1) fängt der Dolmetscher den Satz mit der Konjunktion $m a$ an und wechselt dabei die Perspektive der Rede (im Italienischen ist der Subjekt des Satzes questa situazione), die im Ausgangstext (AT) subjektiv ist (Ich finde, dass...). Im zweiten Beispiel kommt es im Zieltext (ZT) zu Ungenauigkeiten, da es im AT um die Tatsache, dass trotz der höheren Erwerbstätigenquote und der Hochqualifikation vieler Frauen der Anteil weiblicher Führungskräfte unverändert (niedrig) geblieben ist, während die italienische Version eine allgemein niedrige Erwerbstätigenquote angibt (il loro tasso di occupazione è rimasto ugualmente basso).

Als zweite persuasive Strategie gibt Golonka die Verwendung der wir-Form an, weil es den Gemeinschaftssinn anspricht und dabei ein Zusammengehörigkeitsgefühl beim Rezipienten schafft:

2. Ansprechen des „Gemeinschaftssinns“, d. h. Schaffung eines Zusammengehörigkeitsgefühls beim Rezipienten. Typisch ist hier die Verwendung der wir-Form, die den Eindruck von Zugehörigkeit zu derselben sozialen Gruppe schafft. Es wird eine für den Empfänger verständliche und ihm nahe Sprache gebraucht. Häufig sind Appelle an gemeinsame Kategorien, Vorstellungen, Orientierungen, Werte.

(ibidem)

Auch diese Strategie wird in dem Korpus häufig benutzt, da die Abgeordneten immer die wirForm verwenden womit sie nicht nur die Mitglieder ihrer Partei meinen, sondern alle Frauen und alle Mitglieder des Parlaments (und auch alle Bürger der EU), weil das, was sie sagen, zu den akzeptierten Werten der westlichen europäischen Welt gehört. Ein kollektives wir kann aus soziologischer Sicht nur funktionieren, wenn es auch ein kollektives ihr gibt. Diese Abgrenzung realisiert sich dann auch sprachlich durch identitätsstiftende Lexik. Das kollektive ihr umfasst in diesem Fall diejenigen, die sich der Verwirklichung der Gleichberechtigung entgegenstellen. Ziel der Reden ist, die Zuhörer zu überzeugen, dass es immer noch nötig ist, neue Gesetze, neue Reglementierungen für bessere Lebensverhältnisse zu erlassen, die ein familienfreundlicheres Arbeitsumfeld und ein gesellschaftliches Umdenken schaffen. 
DE

(3) Wir haben viele Programme. Frau Ferrero-Waldner, die Kommissarin, hat darauf hingewiesen, dass eben auch sie weltweit regierende Frauen oder Frauen in Regierungsverantwortung angesprochen hat. Das reicht anscheinend nicht.

Es ist nicht nur ein Problem von Frauen, es ist ein Problem von allen Menschen auf der Welt und wir müssen alles tun, um von der Europäischen Union hier etwas zu verbessern.
IT

Eppure noi abbiamo cercato di interpellare le donne a tutti i livelli in tutti le regioni del mondo, donne al potere, questo è insoddisfacente e insufficiente, evidentemente non basta interpellare le donne,

noi come Unione europea dobbiamo fare di tutto per migliorare la situazione.

(Text 4)

In diesem Beispiel referiert Gesine Meissner zum Thema der „Beseitigung von Gewalt gegen Frauen“. In der Verdolmetschung weicht die Perspektive im letzten Satz vom AT ab (,wir müssen alles tun, um von der EU“/,Noi come Unione europea“), weil im AT nicht klar wird, ob die EU verbessert werden soll oder die EU die Situation verbessern soll. Im ZT dagegen wird klar gesagt, dass die EU die Situation der Frauen auf der Welt verbessern soll.

Verwandt mit dieser ist die nächste Strategie:

3. Wahrnehmung der Wirklichkeit in den Kategorien wir/sie und unsere/ihre, d. h. Einteilung der Wirklichkeit in das Eigene und das Fremde.

(ibidem)

Dieser Punkt spielt auch vielleicht auf einen kulturwissenschaftlichen, xenologischen Aspekt an. Im Korpus wird die Wahrnehmung der Wirklichkeit durch Kategorien als Kontrastierung und Polarisierung zwischen der Situation von Männern und jener von Frauen dargestellt (siehe auch das unterstrichene wir im Beispiel [1]):

$\mathrm{DE}$

(4) Und ich denke auch, wir haben es und wir können es uns nicht mehr leisten, das Potenzial von Mädchen, jungen Frauen und Frauen in dieser Gesellschaft derart zu verschenken [...] Aber auf der anderen Seite dürfen wir auch bei einem Gleichstellungsbericht nicht vergessen, auch Männer haben teilweise auch Nachteile. Gerade Jungen haben ganz klar nicht mehr die Möglichkeiten, immer sich in Schulen zu entwickeln. Wir haben auch Defizite im Bereich der Pflege, der Kinderbetreuung; hier können Männer auch aktiv werden.
IT

Dobbiamo, abbiamo investire in queste giovani donne, in queste madri giovani bisogna fare di più e incoraggiare $[\ldots]$ e teniamo presente sempre parlando di questa relazione (...) abbiamo visto che ci sono delle difficoltà per quanto riguarda le scuole e gli uomini forse fanno poco per esempio nella cura dei figli ecc.

Im Vergleich zum AT wird in diesem Beispiel zum Thema Frauenarmut viel stärker zwischen Männern und Frauen polarisiert. Außerdem fällt der Hinweis auf den "Gleichstellungsbedarf" der Männer ganz weg.

Im Folgenden die vierte und letzte von Golonka erwähnte Strategie: 
4. Eine Organisation der kommunizierten Sachverhalte derart, dass der Empfänger sie nur auf eine einzige, nämlich die vom Sender bezweckte Weise interpretieren und verstehen kann. Er wird im Grunde von der Notwendigkeit und Verantwortung eigenständiger Beurteilung und eigener Entscheidungen befreit, indem er auf den einzigen richtigen (den vom Sender erwünschten) Weg zur Lösung des Problems bzw. auf die „einsichtige“ Perspektive der Betrachtung von Wirklichkeit hingewiesen wird.

Golonka (2009: 161-162)

Auch wenn das Publikum der EU-Reden nicht irgendein Publikum ist, sondern es sich um Abgeordnete der EU handelt, wird im Korpus die Notwendigkeit einer neuen Reglementierung als einzige Lösung dargestellt, weil wiederholt betont wird, dass die Gleichstellung nicht nur ein Problem der Frauen, sondern ein Problem von allen Menschen auf der Welt ist und dass man alles tun muss, um in der Gleichberechtigungsfrage etwas zu verbessern (siehe auch Beispiel [3]):

$\mathrm{DE}$

IT

(5) Also Zugang zu Bildung auf jeden Fall, Questo porta a una maggiore sicurezza, anche del Zugang zu Sicherheitssystemen, und dann werden wir auch für die Frauen viele Verbesserungen sistema e questo sicuramente è un miglioramento per le donne. Grazie erreichen!

(Text 5)

(6) Wir wollen nicht erst in hundert Jahren feststellen können, dass dann selbstverständlich Recht der Frauen ist, ihr Leben trotz Kindern unabhängig von männlichen Entscheidungen gestalten zu können!

(Text 7)

Im Beispiel (5), wo die Abgeordnete Gesine Meissner über die Arbeitsbedingungen von Frauen in Europa spricht, schließt die Dolmetscherin ihre Verdolmetschung mit einem Satz, der expliziert, dass sie den Sinn des Textes gar nicht richtig verstanden hat: im deutschen Text appelliert die Rednerin am Ende an Tatsachen, die korrigiert werden sollen, damit die Lage der Frauen besser wird (Zugang zu Bildung und zu Sicherheitssystemen). Dies wird im Italienischen ganz anders (gegenteilig) wiedergegeben ("Questo porta a una maggiore sicurezza, anche del sistema e questo sicuramente è un miglioramento per le donne”). Ähnlich auch im Beispiel (6), wo die Bedeutung des ATs total verdreht wird.

Die bisher besprochenen vier Strategien sollten eigentlich keine großen Schwierigkeiten für den Dolmetscher darstellen, da sie, wie schon erwähnt, noch auf der Ebene des Diskurses anzusiedeln sind. Trotzdem kann man beobachten, dass in einigen Fällen (z. B. [4], [5] und [6]) Probleme in der Verdolmetschung entstehen.

\subsubsection{Die Rolle der Emotionalisierung als persuasive Strategie}

Andere wichtige Kategorien auf diesem Niveau, die auch sprachliche Implikationen mit sich bringen, werden von Schwarz-Friesel in ihrem Buch Sprache und Emotion (2007) identifiziert. Zur Rolle der Emotionalisierung als persuasive Strategie merkt die Autorin Folgendes an: „Empathie und Identifikation gelten als Garant für wirkungsvolle Persuasion. Somit lässt sich die über das Emotionspotenzial realisierte, vom Textproduzenten antizipierte und inten- 
dierte Emotionalisierung als persuasive Strategie beschreiben. Persuasive Strategien sind kommunikative Verfahrensweisen, die spezifisch rezipientenbeeinflussend, d. h. intentional auf eine bestimmte Wirkung ausgerichtet sind" (S. 223). Sie unterscheidet folgende persuasive Strategien für die massenmediale Berichterstattung, die mit Beispielen aus unserem Korpus belegt werden können:

a. auf Autoritäten berufen (z. B. der UN-Sicherheitstrat..., wie der bekannte Politikwissenschaftler...):

$\mathrm{DE}$

(7) Wir haben viele Programme. Frau

Ferrero-Waldner, die Kommissarin, hat darauf hingewiesen, dass eben auch sie weltweit regierenden Frauen oder Frauen in Regierungsverantwortung angesprochen hat.

\section{IT}

Eppure noi abbiamo cercato di interpellare le donne a tutti i livelli in tutti le regioni del mondo, donne al potere, questo è insoddisfacente e insufficiente, ...

In dem in 5.1.1 schon erwähnten Beispiel ist zu beobachten, dass in der italienischen Verdolmetschung die Autorität, die im deutschen Text zitiert wird, fehlt. Das erklärt sich hier nicht mit der allgemeinen Schwierigkeit, beim Hören Namen zu verstehen und zu notieren, da einem EU-Dolmetscher Frau Ferrero-Waldner bekannt sein müsste. Die Auslassung hat wahrscheinlich andere Gründe, die sich allerdings nicht eruieren lassen.

b. auf regelhafte Beziehungen (naturgemäß... traditionell...) und auf kausale Faktoren (als Ursache, zwangsläufig) referieren:

$\mathrm{DE}$

(8) Wir haben einen Bericht zu Frauen und Armut; ein wichtiger Grund dafür ist die Lohnungleichheit zwischen Frauen und Männern. Es wurde schon gesagt: Seit 1957 ist die Lohngleichheit in den Römischen Verträgen verankert. Aber der Lohnunterschied ist heute bei immer noch europaweit bei 17,5\%, in Deutschland gar bei $23 \%$.

\section{IT}

Si è parlato del divario salariale, abbiamo detto che dal '57 la parità a livello di salari è prevista nei Trattati di Roma, però questa differenza ammonta ancora al 17,5\%, in Germania al 23\%.

Auch in diesem Beispiel, ein weiterer Beitrag zum Thema Frauenarmut anlässlich des hundertsten internationalen Tag der Frau, fehlt in der italienischen Wiedergabe nicht nur der Bericht, auf den sich die Rednerin bezieht, sondern auch der Zusammenhang zwischen dem Bericht über Armut und den Gründen dafür.

c. auf Authentizität berufen (z. B. unsere Augenzeugen vor Ort...):

Im Korpus geht es eher um internationale/institutionelle Akten, die die Richtigkeit bestimmter Forderungen rechtfertigen: 
DE

(9) Wir haben den Lissabon-Vertrag jetzt gerade kurz vor der Unterzeichnung, da wird die Menschenrechts-Charta als Annex noch besondere Gültigkeit haben und darum sind wir, denke ich, als Europäische Union besonders verpflichtet, alles zu tun, um diese Gewalt zu bekämpfen.

\section{IT}

Recentemente il, la Carta dei diritti fondamentali è entrata a far parte del Trattato dell'Unione europea, pertanto l'Unione europea deve fare tutto quanto in suo potere per lottare contro questa forma di violenza.

(Text 4)

Hier werden alle Elemente wiedergegeben, es fehlt allerdings die genaue Bezeichnung des Vertrags.

d. Sympathieträger präsentieren (,die kleinen Kinder““, „die junge Mutter“)

$\mathrm{DE}$

(10) Es wurde schon angesprochen, dass auch Gewalt im Krieg, Vergewaltigung als Waffe eingesetzt werden kann und es wurde auch hingewiesen auf den Kongo, wo das seit Jahren passiert in schlimmster Variante, wo auch Vergewaltiger vor kleinen Kindern und vor hochbetagten Frauen nicht Halt machen.
IT

La violenza, lo stupro che in luoghi in conflitto vengono utilizzate come arma. In Congo da anni né le bambine né le donne anziane si possono sentire al sicuro dall'utilizzo di quest'arma.

In diesem Fall schafft es die Dolmetscherin, beide Elemente, Kinder und ältere Frauen, die von den Zuhörern höchstwahrscheinlich als Sympathieträger empfunden werden, in der Verdolmetschung zu bewahren, allerdings mit einigen Modifikationen des Satzes, die den Sinn aber wiedergeben.

e. Atmosphäre über Analogien präsentieren („Wie im Schlaraffenland“, „Wie Dantes Inferno")

DE

(11) Dass wir noch immer über die Gleichstellung von Mann und Frau diskutieren müssen, ist ein absolutes Armutszeugnis unserer Gesellschaft._Frauen leiden unter Benachteiligung, nicht nur in der Arbeitswelt, sondern auch im gesellschaftlichen Leben. Die Liste scheint endlos, daher einige Stichworte: Lohnniveau, Arbeits- und Einstellungsbedingungen, prekäre Arbeitsverhältnisse, Rentenansprüche, Kinderbetreuung, soziale Sicherheit und diverse Formen von Gewalt gegen Frauen.
IT

Che si parli sempre ancora di parità tra uomini donne è veramente una dimostrazione di quanto avviene all'interno della nostra società perché c'è un, uno svantaggio delle donne non soltanto a livello di società, parliamo qui per esempio per quanto riguarda delle condizioni di lavoro, delle condizioni pensionistiche, di quanto riguarda la cura dei bambini, previdenza sociale, tutte le forme di violenza contro le donne.

(Text 9)

In diesem Beispiel fehlt in der Verdolmetschung das Bild des Armutszeugnisses, d. h. das Bild einer Gesellschaft, die unfähig ist, Gerechtigkeit zwischen Mann und Frau zu schaffen. Das Bild des Armutszeugnisses lässt sich aber auch nicht leicht wiedergeben. Es fehlt aber nicht nur das Bild, sondern die negative Bewertung allgemein, die man z. B. mit incapacità della nostra società hätte ausdrücken können. 
f. hervorheben (im positiven oder negativen Sinn) (z. B. „,besonders brutal“, „mit besonderer Vorsicht")

DE

(12) Gewalt gegen Frauen ist ein schreckliches Menschenrechtsverbrechen, egal in welcher Form sie passiert, ob als Zwangsverheiratung, als Schläge, als Vergewaltigung. Besonders schlimm finde ich das Wort „Ehrenmord“, denn das ist überhaupt nicht ehrenhaft. Ehrenmord ist einfach abscheuliches Verbrechen!
IT

La violenza contro le donne è una terribile violazione dei diritti dell'uomo indipendentemente dalla forma assunta, matrimonio coatto, percosse, stupro. In particolare mi riferisco alla...omicidio d'onore che è uno dei crimini più efferati.

(Text 4)

Die hervorgehobenen Elemente werden in diesem Beispiel zwar wiedergegeben, aber in Falle von „Besonders schlimm“ erhält der Ausdruck in der Verdolmetschung einen anderen (semantischen) Wert: Besonders wird nicht als Verstärkung vom Adjektiv schlimm wiedergegeben, sondern als reines Satzdverb.

Dazu kommt eine persuasive Strategie, die meiner Meinung nach für die parlamentarische Rede besonders wichtig ist, und das ist der

g. Appell an die Anwesenden

$\mathrm{DE}$

(13) Frau Reding, Sie haben gesagt, dass die Unternehmen bis nächstes Jahr pledgen müssen, also sich verpflichten müssen, Frauen zu ernennen. Möchten Sie, dass Sie bis nächstes Jahr die pledges haben oder dass sie bis nächstes Jahr Frauen ernannt haben? Ich höre für, dass Sie das Zweitere wollen.
IT

quindi per l'anno prossimo bisogna veramente far sì che siano nominate delle donne. Ma c'è, si vogliono semplicemente delle promesse o si vogliono veramente delle nomine? Io spero che l'obiettivo sia il secondo.

Diese Strategie wird, wie in diesem Beispiel, häufig vom Dolmetscher wegen Zeitmangel, mangelnder Konzentration, Überlastung des Gedächtnisses u. a. ausgelassen.

\subsection{Mikroebene des Diskurses: sprachliche Mittel der Persuasion}

In älteren Arbeiten haben sich die meisten politischen Sprachuntersuchungen besonders intensiv auf das Wort konzentriert (cf. Dieckmann 1975: 106) und die Untersuchung von Texten vernachlässigt. Das resultiert daraus, dass in der politischen Sprache der Wortschatz eine besondere Rolle spielt (Schlagwörter, Slogans, und alle Wörter, die zum politischen Wortschatz gehören). Neuere Studien, wie Schröter/Carius (2009), Ortak (2004), Girnth (2002) u. a., untersuchen aber auch den Stil der Überzeugungsrede. Kontrastive Untersuchungen im Hinblick auf Deutsch-Italienisch gibt es, meines Wissens, keine.

Sprachliche Merkmale befinden sich auf allen vier Ebenen des Sprachsystems: auf phonetischer Ebene (Assonanz, Alliteration, Lautmalerei, Rhythmus, Intonation, Tonfarbe und stärke etc.), auf morphologischer Ebene (Temporalität, Modalität, Genus Verbi, Steigerung der Adjektive, Gebrauch der Interjektionen etc.), auf syntaktischer Ebene (Konnektoren, Hypotaxe, Parataxe, Asyndeton, Polysyndeton, Wortfolge, Satzlänge, Herausstellungen wie 
Linksversetzung [LV] und Rechtsversetzung [RV] usw.), sowie auf lexikalischer Ebene (Fremdwörter, Archaismen, Neologismen, Anachronismen, Dialektismen, Fachwörter, Phraseologismen etc.; cf. Novotná 2008: 11). Ausgewählte Beispiele aus den letzten beiden Bereichen sollen die besonderen Probleme der Übersetzung der Persuasionsstrategien aufzeigen.

\subsubsection{Syntaktische Ebene}

Als Mittel der Persuasion werden bestimmte syntaktische Konstruktionen benutzt, wie z. B. die Linksversetzung oder die Rechtsversetzung, in denen ein satzgliedwertiger Ausdruck mit nominalem Kern (NP, $P P$ ) nach links vor das Vorfeld bzw. nach rechts an das Satzende versetzt wird und damit hervorgehoben wird, wobei der ursprüngliche Platz durch ein pronominales Element markiert ist. Diese Konstruktionen werden nur selten vom Dolmetscher wiedergegeben:

$\mathrm{DE}$

(14) Die Verlängerung

Mutterschutzurlaubs, der die Gesundheit der Mutter im Fokus hat, sie ist nicht nur zum Vorteil für die Frauen, denn Unternehmen werden weniger Frauen einstellen, da Männer diesen Ausfall nicht haben.
IT

Il congedo parentale pone al centro la salute delle donne e questo va a vantaggio delle donne ma anche delle aziende.

Das Beispiel zeigt auch, wie schwierig es ist, nicht nur rhetorische Elemente wiederzugeben, sondern manchmal auch die Intention des Sprechers. Unterstrichen sind hier deshalb Konstituenten, die eine Gegenbedeutung besitzen.

Belegt ist auch die Umwandlung von nicht markierten Sätzen in markierte Sätze:

$\mathrm{DE}$

(15) Und Frauen haben auch die weniger qualifizierten Tätigkeiten.
IT

E poi hanno anche, svolgono anche attività meno qualificate, le donne.

(Text 5)

\subsubsection{Lexikalische Ebene}

Ein Merkmal der politischen Rede ist der Gebrauch von Fachsprache und Fachwörtern, deren Bedeutung eindeutig ist, denn der Leser oder Hörer ,wird nicht mit der Mehrdeutigkeit der Lexeme des allgemeinen Wortschatzes konfrontiert, welche der Argumentation die wünschenswerte Überzeugungskraft nehmen“(Mikulová 2005: 95).

Im vorliegenden Korpus kommen viele Fachwörter vor, die aus verschiedenen Bereichen wie Politik, EU oder spezifischen Themen entliehen werden:

Wortschatz der Politik: ratifizieren, Gesetzgebungskompetenz, Quote, usw.

Wortschatz der EU: Richtlinie, Verordnung, Stellungnahme, Subsidiarität, Ratspräsidentschaft, Berichterstatterin, Kommissionsvorschlag usw.

Wortschatz gesellschaftlicher und wirtschaftlicher Gleichstellung von Männern und

Frauen: Verbesserung der Sicherheit und des Gesundheitsschutzes, Mutterschutz, Elternzeit, 
Kündigungsschutz, Versicherungspflichten, soziale Absicherung, kleinere und mittlere Unternehmen usw.

Die Fachwörter der Politik und der EU stellen im Normalfall kein Problem für die EUDolmetscher dar, weil sie meistens sehr hohe Fachkompetenzen besitzen und oft in diesen Bereichen Erfahrung haben. Größere Probleme weisen Fremdwörter auf, die auch sehr häufig verwendet werden, vor allem Wörter aus dem Englischen:

$\mathrm{DE}$

(16) Vor hundert Jahren haben Frauen das aktive und passive Wahlrecht für sich eingefordert und heute kämpfen wir nach wie vor für die Gleichstellung von Frauen in vielen Bereichen, vor allem in Beruf, Karriere, aber auch hinsichtlich der Bezahlung der geleisteten Arbeit und des Erwerbs von Leistungsansprüchen, insbesondere im Alter und das enthüllt nicht nur das gender pay gap.
IT

Cent'anni fa le donne hanno richiesto il diritto di voto, ora continuiamo a lottare per i diritti della donna e l'uguaglianza di genere per quanto riguarda il mondo del lavoro, le, i diritti di pensionistici e quello che è il salario e non solo

Hier fehlt die Verdolmetschung ganz, wahrscheinlich auch wegen des code-switchings, das hier unerwartet kommt.

Auf lexikalischer Ebene finden wir auch rhetorische Figuren, die zu den Stilelementen gehören und der Expressivität und Ausdrucksvariation dienen. Man unterscheidet die Tropen (semantische Figuren) von den syntaktischen Stilfiguren (cf. Malá 2003: 78). Im Korpus finden wir eine Metapher, ${ }^{13}$ bzw. eine Personifizierung, die eine Nebenform der Metapher ist, die häufig vorkommt, d. h. die Armut als weibliche Figur:

$\mathrm{DE}$

(17) Ich erinnere: 2010 war das Jahr zur Bekämpfung der Armut. Die Armut ist weiblich. Dies hat schwerwiegende Folgen für die Gesellschaft, denn Armut macht krank und kann in der Folge zu Arbeitslosigkeit führen.
IT

Ricordo che c'è stato l'anno di lotta contro la povertà nel 2010 e devo dire che tutto, che però questa povertà può portare ancora di più alla disoccupazione. Grazie.

(Text 9)

In dem ZT wird aber diese Personifizierung nicht erwähnt, wahrscheinlich, weil es nicht als wesentlich empfunden wurde.

Im Gegensatz zu Tropen sind syntaktische rhetorische Figuren an den Satz gebunden. Darunter sind Figuren der Wiederholung - Anapher, Epizeuxis, Parallelismus etc. - zu erwähnen. Unten folgen zwei Beispiele von Parallelismus mit partieller Wiederholung. In beiden Fällen, wie es schon vorauszusehen ist, werden beim Dolmetschen die Wiederholungen vermieden, um Zeit zu sparen.

${ }^{13}$ Zum Problem der Übersetzung von Metaphern und Wiederholungen cf. Schreiber (2012). 
$\mathrm{DE}$

(18) Studien belegen, dass selbst innerhalb dieser Teilzeitjobs Frauen noch dramatisch schlechter bezahlt werden als Männer für die gleiche Zeit, für die gleiche Tätigkeit.

(19) Frauen in die Wirtschaft, Frauen in die Arbeit - so die Forderungen. Wir rufen nach Sonderstellungen, um Elternzeit und Mutterschutz auszugleichen, und wir erreichen damit das Gegenteil: Frauen werden zu teuer und deshalb nicht eingestellt. Alle Maßnahmen sind von der Wirtschaft aus gedacht. Dabei liegt die eigentliche Ungerechtigkeit in der minderwertigen Stellung und in der geringen Anerkennung der Familien- und der hauswirtschaftlichen Tätigkeit.
IT

All'interno di questi lavori part-time le donne sono pagate molto di meno rispetto agli uomini, anche lavorando lo stesso numero di ore.

(Text 7)

[...] Vogliamo far sì che le donne lavorino di più però ci deve essere protezione della maternità, però se diventano madri diventano più costose per questo che vengono lasciate fuori dal posto del lavoro, per questo che hanno qualifiche inferiori e perché le loro attività in casa non vengono riconosciute.

(Text 2)

Im Beispiel (18) hat der Dolmetscher mit dem italienischen Gerundium das Problem der Wiederholung gut gelöst und die Bedeutung gewahrt, aber in dem anderen Beispiel (19) hat die Dolmetscherin große Schwierigkeiten, den Sinn der Wiederholung und des Folgesatzes zu verstehen und daher beide korrekt zu übertragen.

\section{Schluss}

Persuasion wiederzugeben ist für einen Dolmetscher deshalb nicht leicht, weil die Sprachsysteme so verschieden sind, dass es schwer fällt, die gleichen Strategien zu benutzen. Ein zwischensprachlicher Vergleich von Ausgangstexten und ihrer Verdolmetschung seitens der Sprachwissenschaftler sollte daher immer nur Sinneinheiten in Betracht ziehen, weil die Umformulierungen einzelner Teilsätze oft so grundsätzlich verschieden sind, dass die Verdolmetschung kaum noch mit dem Originaltext zu vergleichen ist. Auch wenn die Botschaft oft vermittelt wird, geht beim Dolmetschen häufig nicht nur ein Teil des informativen Sprachpotentials der Ausgangssprache verloren, sondern vor allem das Emotionale an der Sprache, was aber eine große, wenn nicht die größte Rolle bei den Persuasionsstrategien spielt. Das betrifft nicht nur den Ton, die Pausen der Redner usw., sondern auch alle anderen Ausdrucksebenen (Lexik, Syntax, Rhetorik usw.). Die Frage, ob und wie Dolmetscher diese Verluste mit anderen Strategien ausgleichen und beide Elemente - Persuasionsstrategien und Inhalt - beibehalten können, ist noch nicht ausreichend geklärt. Der Dolmetscher selbst könnte zwar auch beispielweise Ton oder Pausen einsetzen, um diese Verluste zumindest teilweise auszugleichen aber aus der Analyse ergibt sich das nicht. Die Dolmetscher behalten manchmal persuasive Elemente bei, verlieren dann aber oft inhaltliche Elemente; oder andersherum: persuasive Elemente werden zugunsten der Übertragung von inhaltlichen Elementen reduziert. Oft ist der Zeitfaktor das entscheidende Element: alle Entscheidungen gehen aus der Notwendigkeit hervor, schnell und knapp zu dolmetschen, und nicht aus dem Prinzip der Schönheit der Sprache. Das führt dann dazu, dass Persuasionsstrategien weggelassen werden, wie oben konstatiert. Die Außenstruktur dieser speziellen Art der Kommunikation wirkt vor allem auf die Wiedergabe von Emotionalität, da durch den kommunikativen Kanal bestimmte Zwänge entstehen. 
In wenigen Fällen sind kulturelle Vorlieben eine mögliche Ursache für die Nicht-Übertragung bestimmter Strategien. Ein Beispiel dafür ist das häufige Weglassen direkter Anreden seitens der Redner (wie im Beispiel [13]), die im Italienischen weniger häufig vorkommt. Aber auch hier könnte es sich um die Notwendigkeit handeln, Zeit zu sparen, in der Hoffnung, dass alle die Anrede auch ohne Verdolmetschung verstehen können.

Zusammenfassend ist in diesem Korpus festzustellen, dass der Dolmetscher bei seiner Arbeit so vielen Problemen gegenübersteht, dass die persuasiven Strategien von ihm vielleicht wahrgenommen aber nicht wiedergegeben werden. Das könnte einerseits daran liegen, dass der Dolmetscher davon ausgeht, dass die nicht transkribierten non verbalen persuasiven Elemente (Lautstärke, Mimik, Gestik usw.) in dem besonderen Kontext der EU-Reden für sich sprechen oder aber dass er die persuasiven Strategien nicht verdolmetschen kann oder will. Im Zentrum steht bei ihm die Botschaft, auch wenn er sie nicht immer "in überzeugender Weise“ überträgt.

\section{Literaturverzeichnis}

Brambilla, Marina (2007): Il discorso politico nei paesi di lingua tedesca: metodi e modelli di analisi linguistica. Roma: Aracne.

Cinato, Lucia (2016): „Ricostruire il senso. Strategie traduttive nell'interpretazione simultanea dal tedesco all'italiano". In: Selig, Maria/Morlicchio, Elda/Dittmar, Norbert (eds.): Gesprächsanalyse zwischen Syntax und Pragmatik. Deutsche und italienische Konstruktionen. Tübingen, Stauffenburg: 245-271.

Cinato, Lucia (2018): „Dinamiche interazionali nella mediazione orale: un'analisi contrastiva tra tedesco e italiano", In: Bermejo Calleja, Felisa/Katelhön, Peggy (eds.): Lingua parlata. Un confronto fra l'italiano e alcune lingue europee. Frankfurt a. M. etc.: Lang: 335-358.

Dieckmann, Walther (1975): Sprache in der Politik. Einführung in die Pragmatik und Semantik der politischen Sprache. Heidelberg: Winter.

Girnth, Heiko (2002): Sprache und Sprachverwendung in der Politik: eine Einführung in die linguistische Analyse öffentlich-politischer Kommunikation. Tubingen: Niemeyer.

Golonka, Joanna (2009): Werbung und Werte. Mittel ihrer Versprachlichung im Deutschen und im Polnischen. Wiesbaden: VS Research.

Gutenberg, Norbert (2000): „Mündlich realisierte schriftkonstituierte Textsorten“. In: Brinker, Klaus et al (eds.), Text- und Gesprächslinguistik. Ein internationales Handbuch. Berlin/New York, de Gruyter: 574-587. (= HSK 16.1).

Kade, Otto (1977): Vermittelte Kommunikation, Sprachmittlung, Translation. Leipzig: Verlag Enzyklopadie.

Lewandowski, Theodor $\left(1994^{6}\right)$ : Linguistisches Wörterbuch. Heidelberg: Quelle \& Meyer. Malá, Jiřina (1993): Einführung in die deutsche Stilistik. Brno: Masarykova univerzita Brno.

Mehrsprachigkeit im Europäischen Parlament. www.europarl.europa.eu/aboutparliament/de/20150201PVL00013/Mehrsprachigkeit [05.07.2019]

Mikulová, Anna (2005): „Diskurs-, Metapher- und Persuasionsproblematik in den publizistischen Texten aus Internet-Seiten, die das Thema der Gesundheit betreffen“. Brünner Beiträge zur Germanistik und Nordistik 19: 85-99. 
Novotná, Viera (2018): Stilistische und persuasive Mittel der politischen Rede, Diplomarbeit, verteidigt am 17.06.2018, Masaryk University in Brno: is.muni.cz/th/179818/ff_b/Bakalarska_praca.pdf [05.07.2019].

Ortak, Nuri (2004): Persuasion. Zur textlinguistischen Beschreibung eines dialogischen Strategiemusters. Tübingen: Niemeyer.

Schreiber, Michael (1993): Übersetzung und Bearbeitung: zur Differenzierung und Abgrenzung des Übersetzungsbegriffs. Tübingen: Narr.

Schreiber, Michael (2012): „Politische Rhetorik im Übersetzungsvergleich. Am Beispiel von Metaphern und Wiederholungen“. In: Di Meola, Claudio (ed.): Akten der 4. Tagung Deutsche Sprachwissenschaft in Italien: Rom, 4.-6. Februar 2010. Frankfurt a. M. etc., Lang: 73-88.

Schröter, Melanie/Björn, Carius (2009): Vom politischen Gebrauch der Sprache. Wort, Text, Diskurs. Eine Einführung. Frankfurt a. M. etc.: Lang.

Schwarz-Friesel, Monika (2007): Sprache und Emotion. Tübingen: Francke.

\section{Corpus}

europarl.europa.eu/plenary/de/debates-video.html [5.07.2019]

Text 1: Gleichbehandlung von Männern und Frauen, die eine selbständige Erwerbstätigkeit ausüben - Verbesserung der Sicherheit und des Gesundheitsschutzes von schwangeren Arbeitnehmerinnen, Wöchnerinnen und stillenden Arbeitnehmerinnen am Arbeitsplatz (Aussprache), Christa Klass 4.5.2009 (6. Wahlperiode).

Text 2: Gleichstellung von Frauen und Männern - 2009 (Aussprache), Christa Klass 8.2.2010 (7. Wahlperiode).

Text 3: Gleichbehandlung von Männern und Frauen, die eine selbständige Erwerbstätigkeit ausüben (Aussprache), Christa Klass 17.5.2010 (7. Wahlperiode).

Text 4: Beseitigung von Gewalt gegen Frauen (Aussprache), Gesine Meissner 25.11.2009 (7. Wahlperiode).

Text 5: Verbesserung der Sicherheit und des Gesundheitsschutzes von schwangeren Arbeitnehmerinnen, Wöchnerinnen und stillenden Arbeitnehmerinnen am Arbeitsplatz - Frauen in prekären Beschäftigungsverhältnissen (Aussprache), Gesine Meissner 18.10.2010 (7. Wahlperiode).

Text 6: Frauenarmut - Gleichstellung von Frauen und Männern - 2010 (Aussprache), Nadja Hirsch 8.3.2011 (7. Wahlperiode).

Text 7: Frauenarmut - Gleichstellung von Frauen und Männern - 2010 - Gabriele Zimmer 8.3.2011 (7. Wahlperiode).

Text 8: Frauenarmut - Gleichstellung von Frauen und Männern - 2010 (Aussprache), Franziska Katharina Brantner 8.3.2011 (7. Wahlperiode).

Text 9: Frauenarmut - Gleichstellung von Frauen und Männern - 2010 (Aussprache), Angelika Werthmann 8.3.2011 (7. Wahlperiode).

Text 10: 100 Jahre Internationaler Tag der Frau - Rebecca Harms 8.3.2011 (7. Wahlperiode). 